\title{
Prefrontal Cortical-Ventral Striatal Interactions Involved in Affective Modulation of Attentional Performance: Implications for Corticostriatal Circuit Function
}

\author{
Anastasia Christakou, Trevor W. Robbins, and Barry J. Everitt \\ Department of Experimental Psychology, University of Cambridge, Cambridge CB2 3EB, United Kingdom
}

\begin{abstract}
Anatomically segregated systems linking the frontal cortex and the striatum are involved in various aspects of cognitive, affective, and motor processing. In this study, we examined the effects of combined unilateral lesions of the medial prefrontal cortex (mPFC) and the core subregion of the nucleus accumbens $(\mathrm{AcbC})$ in opposite hemispheres (disconnection) on a continuous performance, visual attention test [five-choice serial reaction-time task (5CSRTT)]. The disconnection lesion produced a set of specific changes in performance of the 5CSRTT, resembling changes that followed bilateral AcbC lesions while, in addition, comprising a subset of the behavioral changes after bilateral mPFC lesions previously reported using the same task. Specifically, both $\mathrm{mPFC} / \mathrm{AcbC}$ disconnection and bilateral AcbC lesions markedly affected aspects of response control related to affective feedback, as indexed by perseverative responding in the 5CSRTT. These effects were comparable, although not identical, to those in animals with either bilateral AcbC or mPFC/AcbC disconnection lesions. The $\mathrm{mPFC} / \mathrm{AcbC}$ disconnection resulted in a behavioral profile largely distinct from that produced by disconnection of a similar circuit described previously, between the mPFC and the dorsomedial striatum, which were shown to form a functional network underlying aspects of visual attention and attention to action. This distinction provides an insight into the functional specialization of corticostriatal circuits in similar behavioral contexts.
\end{abstract}

Key words: corticostriatal circuits; visual-spatial attention; affective modulation; action control; medial prefrontal cortex; nucleus accumbens core

\section{Introduction}

Prefrontal corticostriatal systems in the rat brain underlie the control of functions as disparate as working memory (Floresco et al., 1999), pavlovian conditioning (Parkinson et al., 2000), and attention (Christakou et al., 2001). Previously, a circuit comprising the medial prefrontal cortex (mPFC) and the dorsomedial striatum was shown to subserve aspects of attention, as indexed by the effects of a disconnection lesion of the circuit in the fivechoice serial reaction-time task (5CSRTT) (Christakou et al., 2001). That procedure spared the acquisition of autoshaping, which, in contrast, is impaired after bilateral nucleus accumbens core (AcbC) lesions (Parkinson et al., 2000). In the present study, we investigated the role of a system linking the MPFC and the AcbC in the 5CSRTT.

Evidence for the dissociation of aspects of performance in the 5CSRTT suggests that they are mediated by several systems that interact in producing optimal behavior. Thus, effects on inhibi-

\footnotetext{
Received April 1, 2003; revised 0ct. 31, 2003; accepted 0ct. 31, 2003.

This work was supported by a Wellcome Trust Program grant (T.W.R., B.J.E.) and was undertaken within the Medical Research Council Center for Behavioral and Clinical Neuroscience. A.C. was supported by the Medical Research Council (research studentship), the British Federation of Women Graduates (BFWG Charitable Fund Award), the Cambridge University Lundgren Fund (Lundgren Research Award), and Peterhouse, Cambridge (Gunn studentship in Physiological Psychology).

Correspondence should be addressed to Dr. Barry J. Everitt, Professor of Behavioral Neuroscience, Department of Experimental Psychology, University of Cambridge, Cambridge CB2 3EB, UK. E-mail: bje30@cam.ac.uk.

DOI:10.1523/JNEUROSCI.0949-03.2004

Copyright $\odot 2004$ Society for Neuroscience $\quad$ 0270-6474/04/240773-08\$15.00/0
}

tory control of premature responding (impulsivity) have been observed in the absence of effects on response accuracy (Cole and Robbins, 1989; Muir et al., 1996; Harrison et al., 1997), and vice versa (Granon et al., 2000, Chudasama et al., 2003).

The dorsal and ventral striatum are functionally dissociable (Robbins et al., 1986; Amalric and Koob, 1987; Cole and Robbins, 1989; Brown and Robbins, 1991; Baunez and Robbins, 1999; Hauber et al., 2000; Rogers et al., 2001). For example, dopamine (DA) depletion of the dorsal, but not the ventral, striatum produced reaction-time deficits (Amalric and Koob, 1987; Carli et al., 1989). D2 receptor antagonist infusions into the ventral striatum did not affect motor readiness (Hauber et al., 2000), whereas unilateral dorsal striatal DA depletion did, without affecting response selection processes (Brown and Robbins, 1991). Dopaminergic lesions of the ventral striatum spared accuracy in the 5CSRTT, despite slowing response latency (Cole and Robbins, 1989). These effects are consistent with the involvement of ventral striatal DA in the modulation of the probability and speed of responding but not in aspects of attentional function (Robbins et al., 1986).

There is evidence for the involvement of glutamatergic input into the AcbC on the impact of affective information on instrumental responding. For example, selective NMDA receptor antagonist administration in the AcbC impaired the acquisition, but not the established performance, of instrumental lever pressing (Kelley et al., 1997). Furthermore, NMDA, but not DA D2, 
receptor blockade in the nucleus accumbens (Acb) interfered with the modulation of reaction-time performance by signals of reward magnitude, when, in intact animals, the speed of responding showed a direct correlation with the signaled value of the reinforcer (Hauber et al., 2000).

Based on such evidence, we hypothesized that disconnection of the $\mathrm{mPFC} / \mathrm{AcbC}$ circuit would produce effects dissociable from those after disconnection of the $\mathrm{mPFC} /$ dorsal striatal circuit described previously (Christakou et al., 2001). We analyzed performance in the 5CSRTT according to the outcome of trials (reward or punishment) and, therefore, the affective value of preceding responses, to test the hypothesis that the $\mathrm{mPFC} / \mathrm{AcbC}$ circuit plays a greater role in reinforcement and affective processing than attentional aspects of performance.

\section{Materials and Methods}

Subjects

The subjects were 37 adult male Lister hooded rats (Charles River, Margate, UK) housed in pairs under a reversed $12 \mathrm{hr}$ light/dark cycle (lights off at 8:30 A.M.). They were allowed ad libitum access to water and food for $7 \mathrm{~d}$ after their arrival to the experimental facility, while they acclimatized to their new environment. After this initial stage, their diet was restricted to $18-20 \mathrm{gm}$ of standard rodent chow per day each, with water provided ad libitum. They reached the target weight of $85-90 \%$ of freefeeding weight in $\sim 7 \mathrm{~d}$; they were monitored for another $3 \mathrm{~d}$ before their behavioral training commenced. All procedures used in this research were undertaken in accordance with the United Kingdom Animals (Scientific Procedures) Act of 1986.

\section{Behavioral procedures}

5CSRTT. The 5CSRTT has been described in detail previously (Carli et al., 1983; Christakou et al., 2001). A significant difference in the programming of the task contingencies in the present study was that perseverative responses were not punished with a "time-out" (TO) period of darkness $(5 \mathrm{sec})$ as previously (Christakou et al., 2001).

Trial structure and main measures. At the beginning of each session, the house light was illuminated and a sucrose pellet $[45 \mathrm{mg}$ precision food pellets (purified rodent diet); Sandown Scientific, Hampton, UK] was delivered in the magazine. On delivery of the reinforcer, the magazine was illuminated and the event was further signaled by the mechanical noise of the pellet dispenser mechanism. Each trial was initiated when the rat opened the magazine panel. After a $5 \mathrm{sec}$ interval [intertrial interval (ITI)], a light came on in one of the five spatial locations (apertures) on the rear wall; the rat had to locate the stimulus and make a nose-poke response in the appropriate hole during or after the stimulus, but within a predefined, "limited hold" (LH) period (standard, $5 \mathrm{sec}$ ). If no response was detected within this $\mathrm{LH}$, the computer counted an omitted trial and the rat was punished with darkness, the TO period, when the house light was switched off for several seconds (standard, $5 \mathrm{sec}$ ). If the response was timely and accurate, a correct response was recorded and a single pellet was delivered in the magazine as a reward. If the response was performed while the target was still illuminated, the light was extinguished and the reward was delivered. Any additional responses in any of the apertures were recorded as perseverative and had no consequence. If the nose poke was in an aperture other than the one in which the stimulus appeared (incorrect response), a TO period followed. Additional responding was again recorded as perseverative. The next trial was initiated by the rat opening the magazine panel, either to collect a reward or after a TO period. The next stimulus was presented after the ITI; if any responses were made during this period, they were recorded as premature, being punished by a TO. Any additional responses during this darkness period reinitiated the TO.

The computerized data-collecting system also recorded any perseverative magazine panel opening (which was not punished) and three measures of response speed. The latency to respond correctly was the time between the offset of the stimulus and the detection of a correct response. Similarly, the latency to respond incorrectly was the time between the offset of the stimulus and an incorrect response. Finally, the magazine

\section{Table 1. Behavioral manipulations in the 5CSRTT}

\begin{tabular}{|c|c|}
\hline \multicolumn{2}{|c|}{ Bilateral AcbC lesion experiment } \\
\hline Baseline & 10 standard postoperative sessions \\
\hline SD & 3 short SD manipulation sessions $(25,15$, and $5 \mathrm{cs})$ \\
\hline \multirow[t]{3}{*}{ ITI } & 2 short (100 and $300 \mathrm{cs})$ \\
\hline & 1 long (1000 cs) ITI sessions \\
\hline & 1 unpredictable ITI session $(100,300,600$ or $1000 \mathrm{cs})$ \\
\hline White noise & $\begin{array}{l}1 \text { session of random white noise interpolation at } 0,250,450 \text {, or } 500 \mathrm{cs} \\
\text { after trial initiation }\end{array}$ \\
\hline \multicolumn{2}{|c|}{$\mathrm{mPFC} / \mathrm{Acb}$ disconnection lesion experiment } \\
\hline Baseline & 10 standard postoperative sessions \\
\hline SD & 1 short $(25 \mathrm{cs})$ and 1 long (200cs) stimulus duration session \\
\hline \multirow[t]{3}{*}{ ITI } & 1 short ITI session (300cs) \\
\hline & 1 long ITI session (1000cs) \\
\hline & 1 unpredictable ITI session $(100,300,500$, or 1000 cs $)$ \\
\hline White noise & $\begin{array}{l}1 \text { session of random white noise interpolation at } 0,250,450 \text {, or } 500 \mathrm{cs} \\
\text { after trial initiation }\end{array}$ \\
\hline
\end{tabular}

Table 2. Stereotaxic coordinates and infusion volumes

\begin{tabular}{llllll}
\hline & AP & L & DV & Volumes & Rates \\
\hline mPFC & +2.4 & $+/-0.6$ & -1.5 & $0.5 \mu \mathrm{l}$ & $0.5 \mu \mathrm{l} / \mathrm{min}$ \\
& +3.1 & $+/-0.5$ & $-3.0,-1.5$ & $0.5 \mu \mathrm{l}$ & $0.5 \mu \mathrm{l} / \mathrm{min}$ \\
& +3.8 & $+/-0.6$ & -1.5 & $0.5 \mu \mathrm{l}$ & $0.5 \mu \mathrm{l} / \mathrm{min}$ \\
AcbC & +1.2 & $+/-1.8$ & -6.3 & $0.3 \mu \mathrm{l}$ & $0.5 \mu \mathrm{l} / \mathrm{min}$ \\
\hline
\end{tabular}

AP, Anterior-posterior; L, lateral; DV, dorsoventral, measured from dura. Volumes refers to the volume of toxin or vehicle solution infused in each placement. Rates refers to the rate of infusion of the toxin. Stereotaxic coordinates are according to Paxinos and Watson (1998).

latency was the time between a correct response and the opening of the panel to collect the reward. Each session lasted $30 \mathrm{~min}$, or until the completion of 100 trials. At the end of each session, all lights were switched off and the animals were returned to their home cages.

Postoperative testing. After full recovery from the operation (7-10 d), the animals were retrained in the standard task (as in the last stage of the training schedule). This testing on the standard task took place during the first 15 postoperative sessions, during which the control animals quickly reached preoperative criteria and all animals established stable performance. These baseline sessions were followed by a series of manipulations of various parameters of the task, relating primarily to the characteristics of the stimulus used to guide the animal's response and to the timing or frequency of events of each trial in a session. These manipulations allowed variations of the attentional demands of the task and are listed in Table 1. Each of the manipulation sessions was preceded by "standard" sessions until the sham animals reached criterion performance (in all cases, only one such session was required). Only significant effects are reported in Results.

\section{Surgery}

Subjects were anesthetized with Avertin, injected intraperitoneally, at an initial dose of $1 \mathrm{ml} / 100 \mathrm{gm}$ body weight. A small additional volume of the anesthetic was administered, if necessary, during the course of the procedure (typically, $0.5 \mathrm{ml}$ per hour after initial administration). The animals were placed in a standard stereotaxic frame (Kopf stereotaxic instruments) fitted with atraumatic ear bars.

The neurotoxin used was quinolinic acid, in a $0.09 \mathrm{~m}$ concentration solution in sterile PBS at $\mathrm{pH} 7.4 ; 0.1 \mathrm{M} \mathrm{NaOH}$ solution was added to increase the $\mathrm{pH}$. The vehicle solution used for the sham animals was sterile $\mathrm{PBS}$ at the same $\mathrm{pH}$. The solutions were administered through a 29-gauge cannula connected via plastic tubing to a $5 \mu \mathrm{l}$ precision microsyringe (Hamilton Bonaduz AG, Bonaduz, Switzerland), which was mounted on an infusion pump. Each subject received the specified volume of either the neurotoxin or the buffer solution infused over $2 \mathrm{~min}$ at each of the placements. After the infusion, the cannula was left in place for $4 \mathrm{~min}$. The stereotaxic coordinates and volumes of the infusions are listed in Table 2. The incisor bar was set at $3.3 \mathrm{~mm}$ below the interaural line.

Thirty-seven rats were used in this study. In experiment 1, 9 animals 

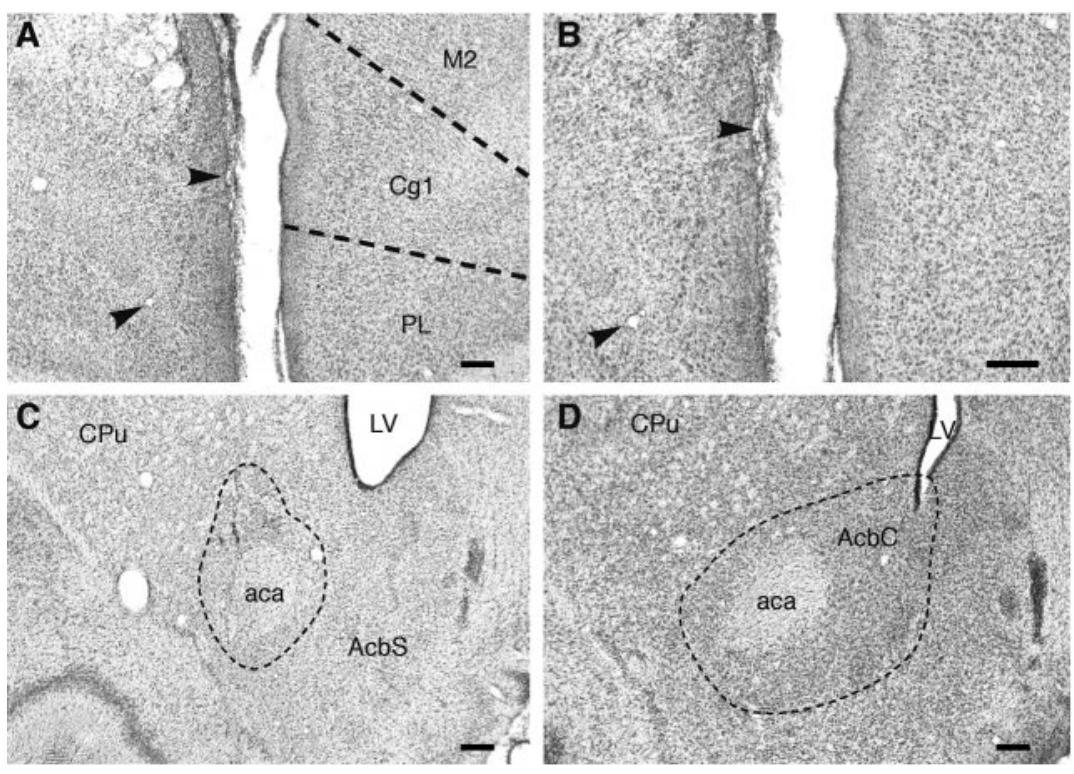

Figure 1. Representative lesion photomicrographs. $A$, Photomicrograph of left unilateral mPFC lesion. $B$, Same lesion as in $A$ but at a higher magnification, with arrowheads indicating recognizable "landmarks" for reference. $C$, Photomicrograph of left AcbC lesion, with dotted line circumscribing the affected area. D, Left sham-operated control AcbC. Scale bars, $100 \mu \mathrm{m}$. aca, Anterior commissure; AcbS, nucleus accumbens shell; Cg1, anterior cingulate cortex; LV, lateral ventricle; M2, motor cortex. ical damage corresponding to the track of the injection cannulae was detectable in most cases. The rostrocaudal extent of the lesions was approximately from 2.0 to 0.5 $\mathrm{mm}$ anterior to the bregma (Paxinos and Watson, 1998). Lesions of the mPFC were unilateral and targeted to the prelimbic (PL) cortex and including parts of cingulate area 1 and infralimbic cortex. No damage to the overlying area Fr2 was observed, apart from the mechanical disruption corresponding to the track of the injection cannulae. The rostral border of the lesions was invariably the emergence of the PL. Caudally, the lesions did not extend anterior to the genu of the corpus callosum, except in two animals that exhibited restricted damage in cingulate area 2. Photomicrographs of representative lesions are shown in Figure 1, $A$ and $B$ (unilateral $\mathrm{mPFC}$ lesion), and Figure 1, $C$ and $D$ (unilateral AcbC lesion and sham, respectively). Figure 2, $A$ and $B$, are schematic representations of the extent of the lesions, in which all lesions have been combined, regardless of laterality.

received bilateral infusions of quinolinic acid solution into the AcbC ("core" group) and 10 received vehicle solution alone in the same coordinates ("sham" group). In experiment 2, eight animals received excitotoxic disconnection of the mPFC and the AcbC ("disc" group), combining unilateral lesions of the $\mathrm{mPFC}$ and the AcbC on opposite sides of the brain, counterbalanced for side. Ten animals served as controls, receiving the appropriate vehicle infusions.

\section{Histology}

Brains were fixed by cardiac perfusion under deep, terminal anesthesia, when $0.01 \mathrm{M}$ PBS was infused for $3 \mathrm{~min}$, followed by a $4 \%$ paraformaldehyde (PFA) solution infusion for $6 \mathrm{~min}$. Both infusions were gravity driven. The brains were removed rapidly, further fixed in PFA for $2 \mathrm{hr}$, and cryoprotected in $20 \%$ sucrose solution overnight, before sectioning on a freezing microtome at $60 \mu \mathrm{m}$ thickness. The sections were mounted on glass slides, dehydrated in an alcohol series, and stained with Cresyl Fast Violet (Raymond Lamb, Eastbourne, UK); they were examined using light microscopy to establish the extent and nature of neuronal damage in the areas infused with excitotoxin, including evidence of gliosis and apparent alterations in ventricular volume.

\section{Statistical analysis}

The SPSS statistical package (release 6.1; SPSS Inc., Chicago, IL) was used for ANOVA (general linear model, type III sum-of-squares). All analyses used full factorial models, with description conforming to standardized notation [dependent variable (performance measure): between-subjects factor (lesion) $\times$ within-subjects factor (session/manipulation)]. The Mauchly sphericity test was used to determine whether the assumption of homogeneity of covariance was violated in a repeated measures analysis of variance design. In cases of violation, the $F$ term was tested against degrees of freedom corrected by the Greenhouse-Geisser epsilon value (the corrected degrees of freedom are reported rounded up to the nearest integer), to give more conservative significance $(p)$ values.

\section{Results}

\section{Histological analysis}

Lesions of the AcbC were highly localized after toxin infusions ventrolaterally within the structure. Damage to the shell subregion of the Acb was detected only in the caudolateral portion of the structure in two of the animals. No excitotoxin-induced damage was observed in the dorsal striatum, although minor mechan-

\section{Behavioral results: bilateral AcbC lesions}

\section{CSRTT measures}

Accuracy, correct latency, and errors of omission. Bilateral core lesions had no effect on accuracy during the baseline sessions (no main effect of lesion on the percentage of correct responses: $\left.F_{(1,17)}=0.20 ; p=0.89\right)$. The latency of the animals to respond correctly was also unaffected by the lesion $\left(F_{(1,16)}=1.50 ; p=\right.$ $0.24)$, as was the percentage of omitted trials $\left(F_{(1,17)}=2.17 ; p=\right.$ $0.16)$.

Premature responding. There was no overall effect of lesion on the level of premature responding $\left(F_{(1,17)}=0.97 ; p=0.34\right)$. When premature responses were divided into those after correct or failed trials (the latter determined by incorrect responses, omissions, and premature nose-pokes), it was shown that there was no effect of lesion on the level of premature responding after correct trials (Fig. 3, top) $\left(F_{(1,13)}=0.07 ; p=0.80\right)$ and that the increase of premature responses after failed trials in the core lesion group relative to the controls (Fig. 3, bottom) was not statistically significant $\left(F_{(1,16)}=3.20 ; p=0.09\right)$. It should be noted that the latencies of premature responses were affected neither by the lesion, nor by the outcome of the previous trial, occurring, on average, $\sim 1$ sec before the end of the ITI.

Perseverative responding. There was a significant main effect of lesion on the overall level of perseverative responding in the core lesion group $\left(F_{(1,17)}=8.27 ; p<0.05\right)$, an increase that persisted for the duration of baseline testing. The pattern of this type of perseveration was such that the difference between the core lesion and the control animals was attributable to perseveration after failed trials $\left(F_{(1,12)}=23.21 ; p<0.001\right)$, whereas there was no effect of lesion on perseveration after correct trials $\left(F_{(1,10)}=1.22\right.$; $p=0.30)$. This comparison is shown in Figure 4 .

In contrast, perseverative panel pushes were elevated in the core lesion group only after correct trials $\left(F_{(1,13)}=7.42 ; p<\right.$ $0.05)$, and not after failed trials $\left(F_{(1,16)}=1.36 ; p=0.26\right)$. This effect was manifested only as a trend when overall perseveration on the magazine panel was analyzed $\left(F_{(1,17)}=3.16 ; p=0.09\right)$. 


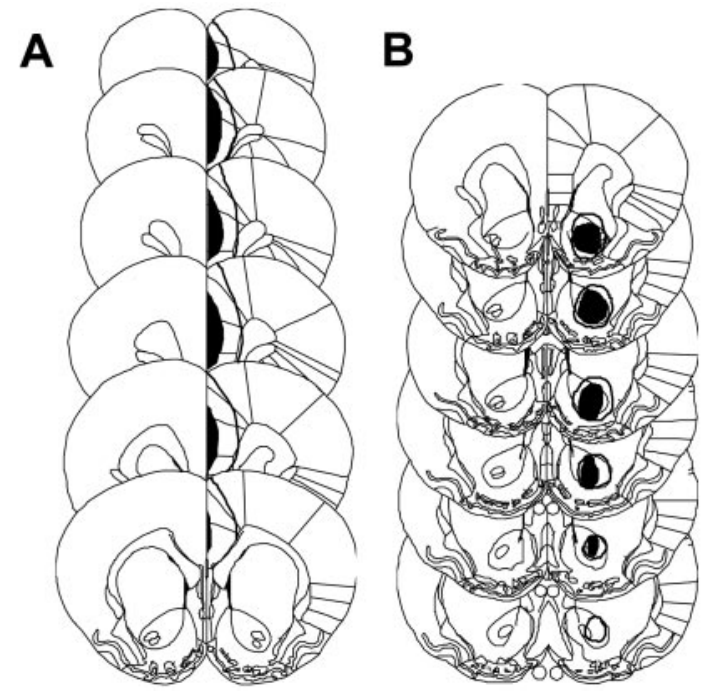

Figure 2. Schematic representation of lesion extent. Schematic representation of the extent of $\mathrm{mPFC}$ lesions $(A)$ and $\mathrm{Acb} C$ lesions ( $B$ ); the extent of the largest (clear) and smallest (black) lesions is depicted. For details on lesion extent, see Results. The schematics were prepared based on the electronic version of Paxinos and Watson (1998).
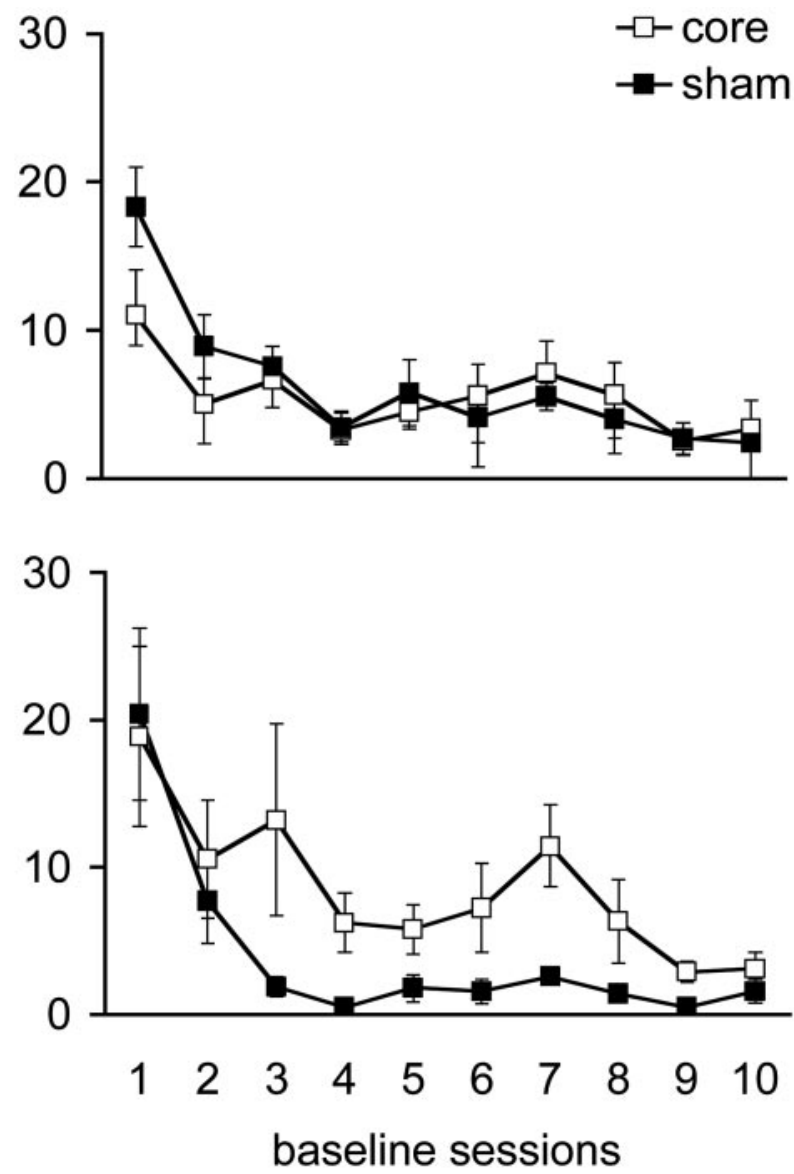

Figure 3. Bilateral $A c b C$ lesions: impulsive responding. The average number of premature nose-pokes performed by animals with bilateral AcbC lesions and their sham-operated controls, within each of 10 baseline sessions, after either correct (top) or failed (bottom) trials is shown. For definition of trial outcomes, see Materials and Methods. core, Bilateral AcbC-lesioned group; sham, bilateral AcbC sham-operated control group.
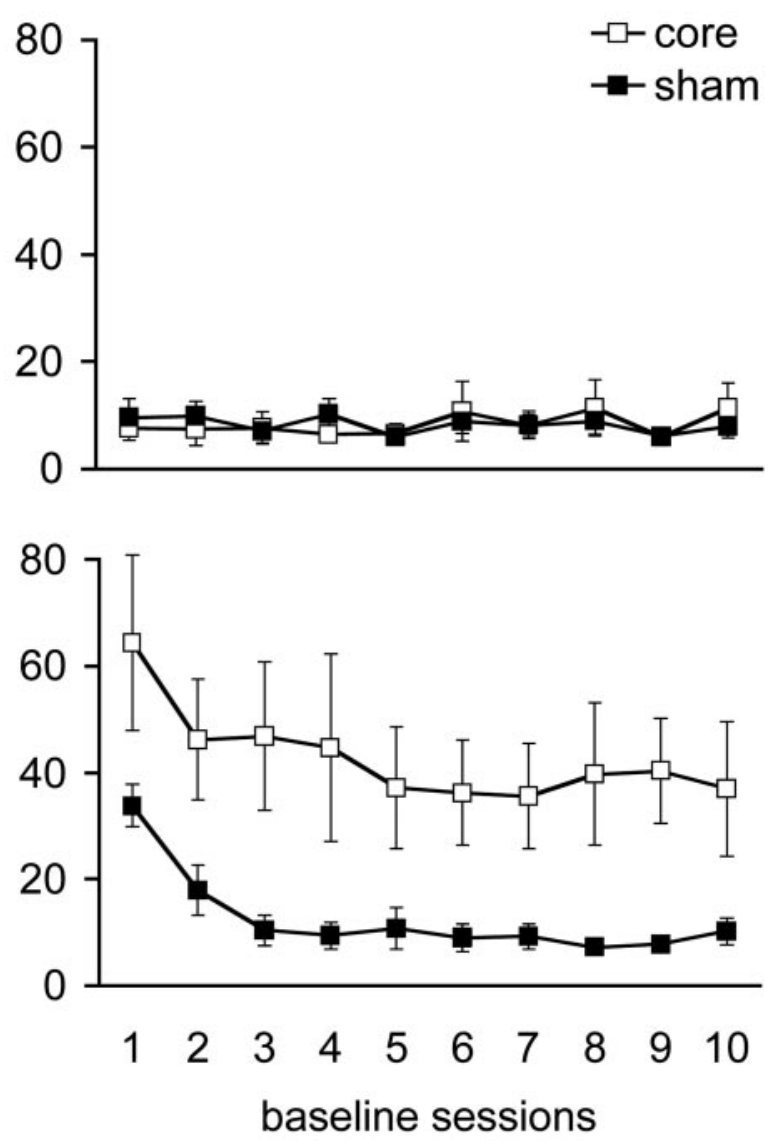

Figure 4. Bilateral $\mathrm{AcbC}$ lesions: compulsive responding. The average number of perseverative nose-pokes performed by animals with bilateral AcbC lesions and their sham-operated controls, within each of 10 baseline sessions, after correct (top) or failed (bottom) trials is shown. For definition of trial outcomes, see Materials and Methods. core, Bilateral AcbClesioned group; sham, bilateral AcbC sham-operated control group.

\section{Behavioral results: $\mathrm{mPFC} / \mathrm{AcbC}$ disconnection lesions} 5CSRTT measures

Accuracy. Disconnection lesioned animals showed an increase in the number of incorrect responses after correct trials (Fig. 5, top) relative to sham-operated controls (main effect of lesion: $F_{(1,14)}=$ $5.85 ; p<0.05)$. In contrast, the number of incorrect responses after failed trials was not affected by the lesion (Fig. 5, bottom) $\left(F_{(1,14)}=0.91 ; p=0.36\right)$. The increase in incorrect responding after correct trials was not attributable to the animals returning to the previously rewarded aperture, because there was no effect of lesion on the ratio of incorrect returns to a previously rewarded aperture over the total incorrect responses after correct trials $\left(F_{(1,16)}=0.01 ; p=0.94\right)$.

Manipulating the stimulus duration (SD) had no differential effect on the number or percentage of correct responses of the two groups, because they both showed equivalent increases in accuracy as the duration of the stimulus was lengthened. However, the disconnection lesioned group, in a pattern similar to that during baseline testing, performed more incorrect responses after correct trials than their sham-operated controls (Fig. 5, top, inset) $\left(F_{(1,16)}=7.58 ; p<0.05\right)$, whereas there was no difference between the groups in the same measure after failed trials (Fig. 5, bottom, inset $)\left(F_{(1,16)}=0.05 ; p=0.82\right)$. The number of incorrect responses after both correct and failed trials was sensitive to changes in the duration of the stimulus (main effect of SD: after correct: $F_{(1,16)}=18.59, p<0.01$; after failed: $F_{(1,16)}=45.82, p<$ 

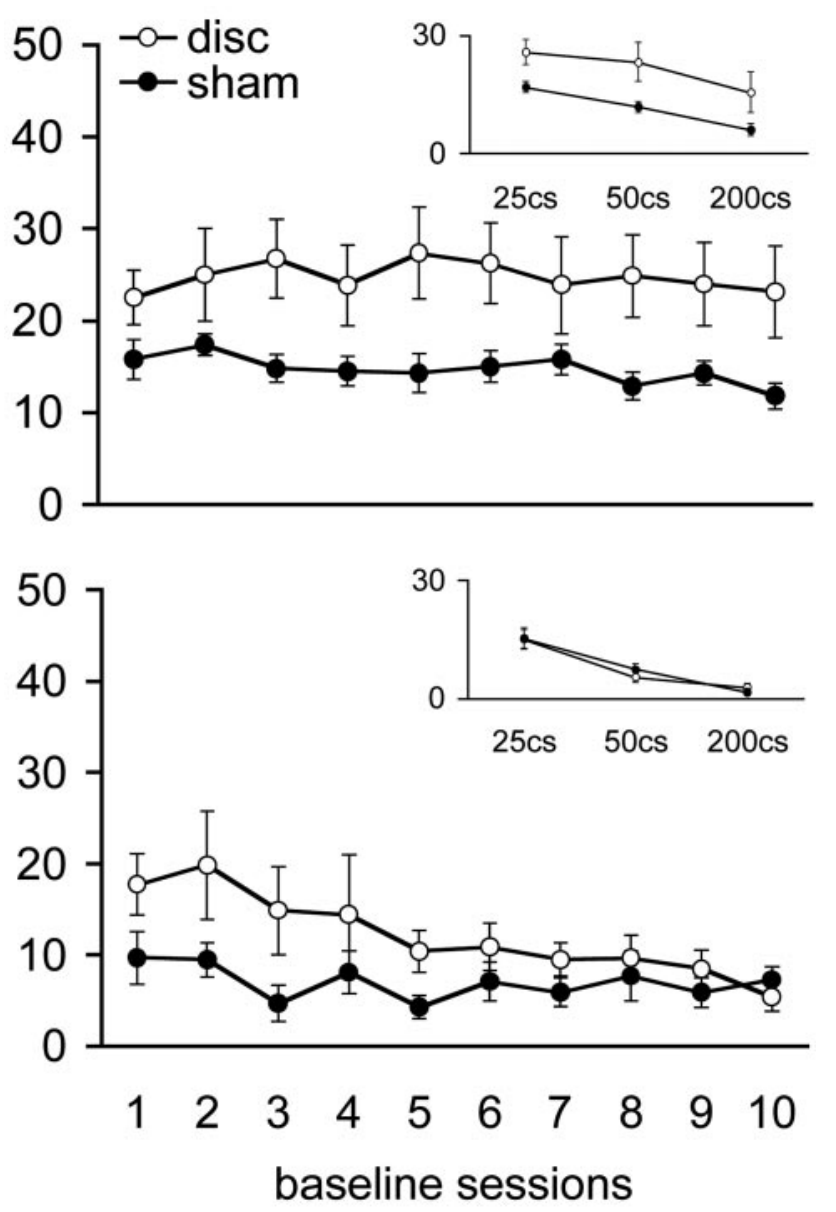

Figure 5. $\mathrm{mPFC} / \mathrm{AcbC}$ disconnection lesions: errors of commission. The average number of incorrect responses to the presentation of the target relative to the outcome of the previous trial, within each of 10 baseline sessions or during two SD manipulation sessions, is shown. Top, Incorrect responses after correct trials during baseline testing and during (inset) the 25 and 200 csec SD sessions (a baseline session at 50csec SD was interpolated and is also shown). Bottom, Similarly, incorrect responses after failed trials during baseline testing and during (inset) the same SD manipulation sessions. For definition of trial outcomes, see Materials and Methods. disc, mPFC/AcbC disconnection lesioned group; sham, mPFC/AcbC disconnection sham-operated control group.

0.001), exhibiting a dramatic decrease with increasing SD. Neither of these effects of stimulus duration interacted with lesion group (after correct: $F_{(1,16)}=0.01, p=0.93$; after failed: $F_{(1,16)}=$ $0.16, p=0.69)$.

Correct latency. The disconnection lesion increased the average latency of correct responding during baseline testing (main effect of lesion: $F_{(1,14)}=26.78$; $p<0.001$ ). The latencies of correct responses that followed either a correct or a failed trial were analyzed separately. In the case of correct responses after a correct trial, lesioned animals were shown to be slower than controls (main effect of lesion: $F_{(1,12)}=29.78 ; p<0.001$ ). There was an initial increase in the latency of the lesioned group to perform a correct response after failed trials (main effect of lesion: $F_{(1,14)}=$ 6.39, $p<0.05$; main effect of session: $\left.F_{(4,55)}=3.44, p<0.05\right)$. However, this measure quickly recovered to control levels, because a session by lesion interaction detected $\left(F_{(4,55)}=2.95 ; p<\right.$ $0.05)$ was attributable to the complete alleviation of the difference between the two groups from session 4 onward (where for sessions 1,2 , and 3, $p<0.01, p<0.05$, and $p<0.01$, respectively).

Premature responding. Premature responding was increased by the lesion $\left(F_{(1,14)}=4.80 ; p<0.05\right)$. However, there was no
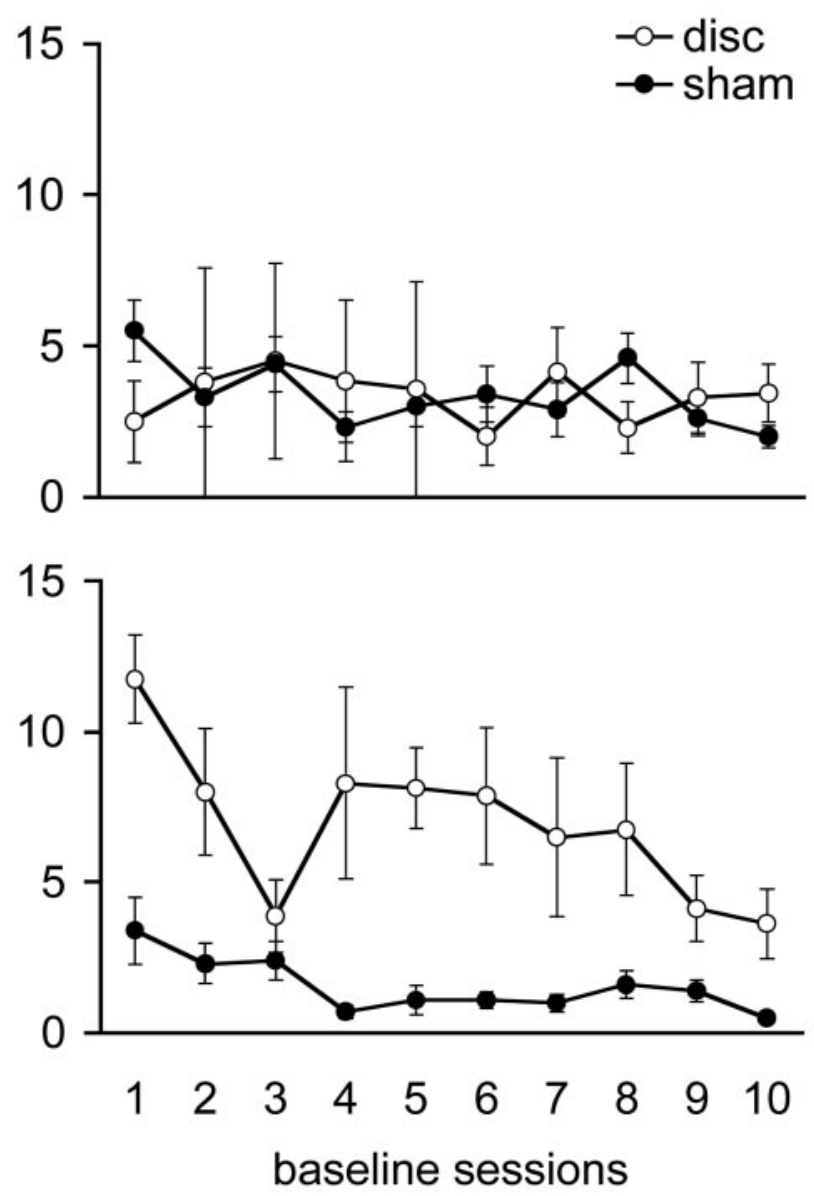

Figure 6. $\mathrm{mPFC} / \mathrm{Acb}$ disconnection lesions: impulsive responding. The average number of premature nose-pokes performed by animals with $\mathrm{mPFC} / \mathrm{Acb}$ C disconnection lesions and their sham-operated controls, within each of 10 baseline sessions, after correct (top) or failed (bottom) trials is shown. For definition of trial outcomes, see Materials and Methods. disc, mPFC/ $A c b C$ disconnection lesioned group; sham, $\mathrm{mPFC} / \mathrm{AcbC}$ disconnection sham-operated control group.

effect of lesion on the level of premature responding after correct trials (Fig. 6, top) $\left(F_{(1,12)}=0.03 ; p=0.87\right)$. Therefore, as can be seen in Figure 6 (bottom), the overall effect of lesion on premature responding was wholly attributable to the difference between the groups only after failed trials $\left(F_{(1,12)}=28.01 ; p<0.001\right)$. The latencies of premature responses were unaffected by the lesion and by the outcome of the preceding trial. On average, premature responses were committed $\sim 1$ sec before the scheduled termination of the ITI, and their distribution relative to the outcome of the previous trial was unaffected by any of the behavioral manipulations.

Perseverative responding. Perseveration in the apertures was also increased by the lesion $\left(F_{(1,14)}=19.29 ; p<0.01\right)$. The level of perseveration on the apertures after correct trials (Fig. 7, top) was not affected by the lesion $\left(F_{(1,13)}=0.10 ; p=0.76\right)$. In contrast, perseverative responses in the apertures after failed trials (Fig. 7, bottom) were significantly elevated in the lesioned group $\left(F_{(1,14)}=27.29 ; p<0.001\right)$.

Perseveration on the magazine panel was not affected by the lesion $\left(F_{(1,14)}=1.83 ; p=0.20\right)$, regardless of the outcome of the previous trial (after correct trials: $p=0.81$; after failed trials: $p=$ $0.14)$. There was also no effect of lesion on panel latency $\left(F_{(1,14)}=\right.$ $0.69 ; p=0.42$ ).

All behavioral results are summarized in Table 3 . 

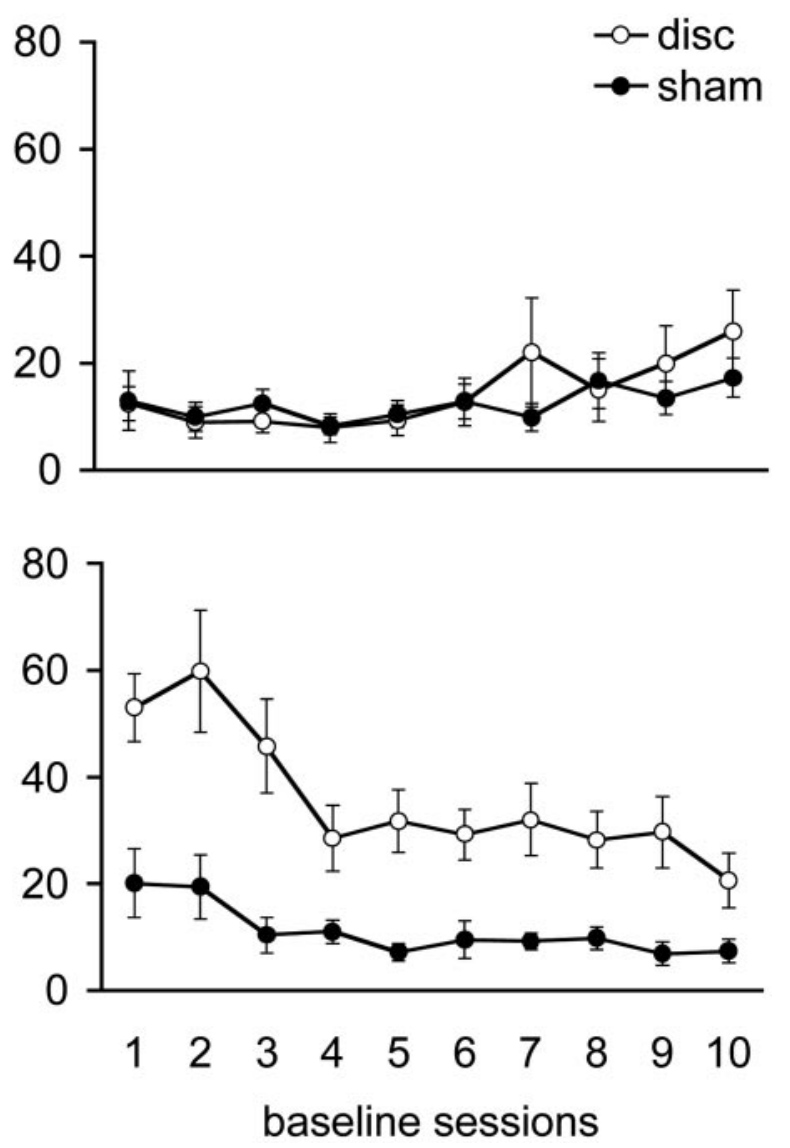

Figure 7. $\mathrm{mPFC} / \mathrm{Acb}$ disconnection lesions: compulsive responding. The average number of perseverative nose-pokes performed by animals with $\mathrm{mPFC} / \mathrm{Acb}$ C disconnection lesions and their sham-operated controls, within each of 10 baseline sessions, after correct (top) or failed (bottom) trials is shown. For definition of trial outcomes, see Materials and Methods. disc, $\mathrm{mPFC} / \mathrm{Acb}$ disconnection lesioned group; sham, mPFC/AcbC disconnection sham-operated control group.

\section{Discussion}

Bilateral lesions of the AcbC produced a distinctive set of impairments in the 5CSRTT, contrasting with those produced by bilateral lesions of the dorsomedial or dorsolateral striatum (Rogers et al., 2001). Unlike damage to the dorsal striatum, bilateral AcbC lesions had no effect on attentional accuracy but affected variables reflecting the inhibitory control of responding, in particular, perseverative behavior (compulsive, returning to the same response) and, to a lesser (nonsignificant) extent, premature behavior (impulsive, occurring prematurely during the delay to reinforcement) (Evenden and Ryan, 1999; Cardinal et al., 2001; Winstanley et al., 2003). These deficits were only apparent as a function of the outcome of the previous trial (i.e., either after TO or positive reinforcement), suggesting that the AcbC is part of circuitry especially implicated in the modulation of attentional performance by affective outcome.

Existing data support the involvement of the Acb (especially the AcbC) in behavioral inhibition, including both impulsive and compulsive forms of responding. For example, lesions of the Acb impair responding in extinction (Reading et al., 1991), under progressive ratio schedules (Bowman and Brown, 1998), in discriminated approach involving pavlovian go-no-go contingencies (Parkinson et al., 1999) and in a delayed gratification procedure for the measurement of impulsive responding (Cardinal et al., 2001). In the present experiment, AcbC lesions produced,
Table 3. Summary of behavioral results

\begin{tabular}{|c|c|c|c|c|}
\hline Manipulation & Baseline & SD & ITI & Noise \\
\hline \multicolumn{5}{|l|}{ Bilateral AcbC lesions } \\
\hline \multicolumn{5}{|l|}{ Analysis of main effects } \\
\hline Accuracy & - & - & - & - \\
\hline Correct latency & - & - & - & - \\
\hline Premature responding & - & $\Uparrow$ & 个 & - \\
\hline Perseverative responding & $\Uparrow$ & $\Uparrow$ & $\Uparrow$ & $\Uparrow$ \\
\hline Magazine perseveration & - & $\Uparrow$ & - & - \\
\hline \multicolumn{5}{|c|}{ Analysis according to the outcome of the previous trial } \\
\hline \multicolumn{5}{|l|}{ Premature responding } \\
\hline After correct & - & - & - & - \\
\hline After failed & $\sim \Uparrow$ & $\Uparrow$ & 个 & - \\
\hline \multicolumn{5}{|l|}{ Perseverative responding } \\
\hline After correct & - & - & - & - \\
\hline After failed & $\Uparrow$ & $\Uparrow$ & $\Uparrow$ & $\Uparrow$ \\
\hline \multicolumn{5}{|l|}{ Panel perseveration } \\
\hline After correct & $\Uparrow$ & $\Uparrow$ & - & - \\
\hline After failed & - & - & - & - \\
\hline \multicolumn{5}{|l|}{$\mathrm{mPFC}-\mathrm{Acb}$ disconnection lesions } \\
\hline \multicolumn{5}{|l|}{ Analysis of main effects } \\
\hline Accuracy & $\Downarrow$ & - & - & - \\
\hline Correct latency & $\Uparrow$ & $\Uparrow$ & $\Uparrow$ & $\Uparrow$ \\
\hline Premature responding & $\Uparrow$ & - & $\Uparrow$ & - \\
\hline Perseverative responding & $\Uparrow$ & $\Uparrow$ & - & - \\
\hline Magazine perseveration & - & - & - & - \\
\hline \multirow{2}{*}{\multicolumn{5}{|c|}{$\begin{array}{l}\text { Analysis according to the outcome of the previous trial } \\
\text { Incorrect responses }\end{array}$}} \\
\hline & & & & \\
\hline After correct & $\Uparrow$ & $\Uparrow$ & $\Uparrow$ & $\Uparrow$ \\
\hline After failed & - & - & - & - \\
\hline \multicolumn{5}{|l|}{ Correct latency } \\
\hline After correct & $\Uparrow$ & $\Uparrow$ & $\sim \Uparrow$ & $\Uparrow$ \\
\hline After failed & - & - & - & - \\
\hline \multicolumn{5}{|l|}{ Premature responding } \\
\hline After correct & - & - & - & - \\
\hline After failed & $\Uparrow$ & $\Uparrow$ & $\Uparrow$ & $\sim \Uparrow$ \\
\hline \multicolumn{5}{|l|}{ Perseverative responding } \\
\hline After correct & - & - & - & - \\
\hline After failed & $\Uparrow$ & $\Uparrow$ & - & - \\
\hline \multicolumn{5}{|l|}{ Magazine perseveration } \\
\hline After correct & - & - & - & - \\
\hline After failed & - & - & - & $\Uparrow$ \\
\hline
\end{tabular}

Summary of the effects after bilateral $\mathrm{Acb} C$ lesions or $\mathrm{MPFC} / \mathrm{AcbC}$ lesions on the main measures of performance on the 5CSRTT. Symbols indicate change (or not) relative to the performance of control animals in the same manipulation.

after failed trials only, significant increases in two forms of perseverative responding, with a trend also for increased premature responses.

In the 5CSRTT, failed trials are signaled by a period of darkness (TO). A failure to integrate the significance of the TO in the behavioral sequence might lead to perseveration on preestablished behavioral schemes (responding to the apertures). Normal rats readily learn the significance of the TO, as indexed by the slowing of acquisition of criterion performance when the TO is omitted and by the tendency to suppress perseverative responding on the apertures after TO periods, turning instead to the reinforcer panel to initiate the next trial (our unpublished observations). Thus, a functional disruption expressed by increased premature and perseverative responding may be related to the location of the TO in the trial. The TO does not relate to the delivery of the primary reinforcer directly but, nevertheless, carries information about the schedule of reinforcement. Specifically, its onset reliably predicts delay of the next opportunity to respond for reinforcement (and consequently signals delay of reinforcement), whereas its offset signals the presence of such an 
opportunity. This information may, therefore, have failed to be integrated in the response schedule of AcbC-lesioned animals, which consequently perseverate on initiated behaviors.

The level and type of disinhibition produced by bilateral AcbC lesions was comparable with that after $\mathrm{mPFC/AcbC}$ disconnection lesions and forms a subset of the deficits that follow bilateral mPFC lesions in the 5CSRTT (Muir et al., 1996). This suggests that the observed disinhibitory effect was mediated by the disruption of the $\mathrm{mPFC} / \mathrm{AcbC}$ system.

The pattern of effects after the mPFC/AcbC disconnection lesion is dissociable from that following the disconnection of an equivalent system linking the $\mathrm{mPFC}$ with the dorsomedial striatum [medial caudate putamen $(\mathrm{mCPu})]$, which has been shown to affect aspects of attentional function in the same task (Christakou et al., 2001). First, increased premature responding after the $\mathrm{mPFC} / \mathrm{mCPu}$ disconnection was shown to be the consequence of an executive deficit in visual attention rather than of disinhibition, whereas this was not the case after the $\mathrm{mPFC} / \mathrm{AcbC}$ disconnection lesions, as evidenced by the fact that both compulsive and impulsive responding only followed trials with negative outcome. This distinction suggests that increases in such responding were consequences of nonreward.

Second, although the mPFC/AcbC disconnection lesion did affect the number of incorrect responses and the speed of correct responses under certain conditions, these were not attributable to impairment in attentional function. Specifically, increases in incorrect responding were observed only after correct, but not failed, trials. Similarly, decreases in the speed of correct responding were observed only for responses after correct, but not failed, trials. It would have been expected that increasing the duration of the stimulus would have (at least partially) alleviated these effects were they related to target detection. However, they were present throughout behavioral testing, proving inflexible to manipulations of stimulus discriminability and persisting despite a complete recovery of overall accuracy and response speed levels, suggesting that they were not caused by an impairment in discrimination or response execution. Furthermore, these deficits are unlikely to have been attributed solely to either of the lesions combined to produce the disconnection; despite significant accuracy deficits after bilateral mPFC lesions in the 5CSRTT (Muir et al., 1996), unilateral mPFC lesions have no appreciable effects (Christakou et al., 2001). Furthermore, as shown in the present study, even bilateral lesions of the AcbC affected neither accuracy nor speed in the task. In addition, we have previously examined the effect of disconnection lesions between the anterior cingulate cortex and the AcbC (our unpublished observations), which had no effect on any aspect of performance in the 5CSRTT, despite marked effects on autoshaping, demonstrating that unilateral lesions of the AcbC do not affect performance in the 5CSRTT. Notably, unilateral lesions of the AcbC do not affect autoshaping, which is severely affected after bilateral lesions of the structure (Parkinson et al., 2000).

Aiding the interpretation of these observations may be the fact that bilateral AcbC lesions also elevated perseveration on the magazine panel after correct trials, an effect that was not observed in the disconnection group. Evidently, reinforcement affected the control of behavior in distinct ways in the two lesioned groups. It is likely that the reinforcing event on completion of a correct trial was inappropriately incorporated into the schedule of responding of the AcbC-lesioned group, delaying the switch from responding on the panel to attending to the apertures. In contrast, although the unilateral AcbC lesions in the disconnection manipulation were insufficient to affect response switching in such a way, their combination with contralateral mPFC lesions (i.e., the disruption of the mPFC-AcbC circuit) interfered with scheduling subsequent responding to the target, leading to elevation of incorrect responding and response latency after reinforcement.

The differentiation of effects after either disconnection or bilateral AcbC lesions (or, indeed, bilateral mPFC lesions) (Muir et al., 1996) suggests that interactions of the mPFC/AcbC system with other brain regions are important in the context of the 5CSRTT and helps identify the neural mechanisms that may underlie the psychological phenomena described above. Frontal and limbic projections onto the Acb are known to form complex interactions and to regulate mesolimbic DA release in the structure (Moore et al., 1999). For instance, the PFC exerts inhibitory control over Acb DA release during amygdala activation (Jackson and Moghaddam, 2001). The PFC may, thus, modulate the DAdependent potentiation by affective information reaching the Acb from the limbic forebrain. This modulation may not only affect Acb output to motor structures but may feed back into the PFC via the thalamus, providing online integration of affective information into the function of other corticostriatal systems. The absence of this modulation in AcbC-lesioned animals may account for the disinhibition of responding, depending on the reinforcing outcome of trials.

This interpretation fits data implicating the Acb and the AcbC, in particular, in assigning the level of incentive value to environmental cues or events (Everitt and Robbins, 1992). Neurons in the ventral striatum code the expectancy of reward (Apicella et al., 1991; Schultz et al., 1992; Bowman et al., 1996) and show differential responses to cues that predict reward or nonreward (Bowman et al., 1996). More importantly, the pattern of activity of ventral striatal neurons has been shown to correlate with the progress through a reinforcement schedule toward a rewarded trial (Shidara et al., 1998). This correlation suggests that the ventral striatum is involved in tracking the progress toward a desired goal within complex behavioral sequences, a process that may be involved in guiding the switch between response programs in the 5CSRTT according to the outcome of trials and the conditioned stimuli that signal such outcomes.

The results of these experiments, considered in conjunction with those from previous studies (Muir et al., 1996; Christakou et al., 2001; Rogers et al., 2001), provide evidence for the functional dissociation of two corticostriatal systems that involve the mPFC and two distinct striatal target regions of the projections from this area, the $\mathrm{mCPu}$ and the AcbC. Specifically, these studies suggest the involvement of the $\mathrm{mPFC} / \mathrm{mCPu}$ system in aspects of executive attention to action and the involvement of the mPFC/AcbC system in the integration of information about the consequences of action in relation to anticipated reward. This dissociation is in line with previous evidence for the involvement of individual nodes of frontostriatal systems in motivated behavior. Furthermore, it provides insight into the way that the PFC can be involved in different aspects of such behavior (namely response organization and the integration of motivational information), as well as in exerting influence on, and receiving indirect modulation from, distinct striatal regions.

\section{References}

Amalric M, Koob GF (1987) Depletion of dopamine in the caudate-nucleus but not in nucleus-accumbens impairs reaction-time performance in rats. J Neurosci 7:2129-2134.

Apicella P, Ljungberg T, Scarnati E, Schultz W (1991) Responses to reward in monkey dorsal and ventral striatum. Exp Brain Res 85:491-500.

Baunez C, Robbins TW (1999) Effects of dopamine depletion of the dorsal 
striatum and further interaction with subthalamic nucleus lesions in an attentional task in the rat. Neuroscience 92:1343-1356.

Bowman EM, Brown VJ (1998) Effects of excitotoxic lesions of the rat ventral striatum on the perception of reward cost. Exp Brain Res 123:439-448.

Bowman EM, Aigner TG, Richmond BJ (1996) Neural signals in the monkey ventral striatum related to motivation for juice and cocaine rewards. J Neurophysiol 75:1061-1073.

Brown VJ, Robbins TW (1991) Simple and choice reaction-time performance following unilateral striatal dopamine depletion in the rat. Impaired motor readiness but preserved response preparation. Brain 114:513-525.

Cardinal RN, Pennicott DR, Sugathapala CL, Robbins TW, Everitt BJ (2001) Impulsive choice induced in rats by lesions of the nucleus accumbens core. Science 292:2499-2501.

Carli M, Robbins TW, Evenden JL, Everitt BJ (1983) Effects of lesions to ascending noradrenergic neurones on performance of a 5-choice serial reaction task in rats; implications for theories of dorsal noradrenergic bundle function based on selective attention and arousal. Behav Brain Res 9:361-380.

Carli M, Jones GH, Robbins TW (1989) Effects of unilateral dorsal and ventral striatal dopamine depletion on visual neglect in the rat: a neural and behavioural analysis. Neuroscience 29:309-327.

Christakou A, Robbins TW, Everitt BJ (2001) Functional disconnection of a prefrontal cortical-dorsal striatal system disrupts choice reaction time performance: implications for attentional function. Behav Neurosci 115:812-825.

Chudasama Y, Passetti F, Desai A, Rhodes S, Lopian D, Robbins TW (2003) Dissociable aspects of performance in the 5 choice serial reaction time task following lesions of the dorsal anterior cingulate, infralimbic and orbitofrontal cortex in the rat: different effects on selectivity, impulsivity and compulsivity. Behav Brain Res 146:105-119.

Cole BJ, Robbins TW (1989) Effects of 6-hydroxydopamine lesions of the nucleus accumbens septi on performance of a 5-choice serial reactiontime task in rats - implications for theories of selective attention and arousal. Behav Brain Res 33:165-179.

Evenden JL, Ryan CN (1999) The pharmacology of impulsive behaviour in rats VI: the effects of ethanol and selective serotonergic drugs on response choice with varying delays of reinforcement. Psychopharmacology 146:413-421.

Everitt BJ, Robbins TW (1992) Amygdala-ventral striatal interactions and reward-related processes. In: The amygdala: neurobiological aspects of emotion, memory, and mental dysfunction (Aggleton JP, ed), pp 401429. New York: Wiley-Liss.

Floresco SB, Braaksm DN, Phillips AG (1999) Thalamic-cortical-striatal circuitry subserves working memory during delayed responding on a radial arm maze. J Neurosci 19:11061-11071.

Granon S, Passetti F, Thomas KL, Dalley JW, Everitt BJ, Robbins TW (2000) Enhanced and impaired attentional performance after infusion of D1 dopaminergic receptor agents into rat prefrontal cortex. J Neurosci 20:1208-1215.

Harrison AA, Everitt BJ, Robbins TW (1997) Central 5-HT depletion en- hances impulsive responding without affecting the accuracy of attentional performance: interactions with dopaminergic mechanisms. Psychopharmacology 133:329-342.

Hauber W, Bohn I, Giertler C (2000) NMDA, but not dopamine D-2, receptors in the rat nucleus accumbens are involved in guidance of instrumental behavior by stimuli predicting reward magnitude. J Neurosci 20:6282-6288.

Jackson ME, Moghaddam B (2001) Amygdala regulation of nucleus accumbens dopamine output is governed by the prefrontal cortex. J Neurosci 21:676-681.

Kelley AE, SmithRoe SL, Holahan MR (1997) Response-reinforcement learning is dependent on $\mathrm{N}$-methyl-N-aspartate receptor activation in the nucleus accumbens core. Proc Natl Acad Sci USA 94:12174-12179.

Moore H, West AR, Grace AA (1999) The regulation of forebrain dopamine transmission: relevance to the pathophysiology and psychopathology of schizophrenia. Biol Psychiatry 46:40-55.

Muir JL, Everitt BJ, Robbins TW (1996) The cerebral cortex of the rat and visual attentional function: dissociable effects of mediofrontal, cingulate, anterior dorsolateral, and parietal cortex lesions on a five-choice serial reaction time task. Cereb Cortex 6:470-481.

Parkinson JA, Olmstead MC, Burns LH, Robbins TW, Everitt BJ (1999) Dissociation in effects of lesions of the nucleus accumbens core and shell on appetitive pavlovian approach behavior and the potentiation of conditioned reinforcement and locomotor activity by $\mathrm{D}$-amphetamine. J Neurosci 19:2401-2411.

Parkinson JA, Willoughby PJ, Robbins TW, Everitt BJ (2000) Disconnection of the anterior cingulate cortex and nucleus accumbens core impairs pavlovian approach behavior: Further evidence for limbic cortical-ventral striatopallidal systems. Behav Neurosci 114:42-63.

Paxinos G, Watson C (1998) The rat brain in stereotaxic coordinates, Ed 4. London: Academic.

Reading PJ, Dunnett SB, Robbins TW (1991) Dissociable roles of the ventral, medial and lateral striatum on the acquisition and performance of a complex visual stimulus-response habit. Behav Brain Res 45:147-161.

Robbins TW, Evenden JL, Ksir C, Reading P, Wood S, Carli M (1986) The effects of D-amphetamine, alpha-flupentixol, and mesolimbic dopamine depletion on a test of attentional switching in the rat. Psychopharmacology 90:72-78.

Rogers RD, Baunez C, Everitt BJ, Robbins TW (2001) Lesions of the medial and lateral striatum in the rat produce dissociable deficits in attentional performance. Behav Neurosci 115:799-811.

Schultz W, Apicella P, Scarnati E, Ljungberg T (1992) Neuronal activity in monkey ventral striatum related to the expectation of reward. J Neurosci 12:4595-4610.

Shidara M, Aigner TG, Richmond BJ (1998) Neuronal signals in the monkey ventral striatum related to progress through a predictable series of trials. J Neurosci 18:2613-2625.

Winstanley CA, Chudasama Y, Dalley JW, Theobald DE, Glennon JC, Robbins TW (2003) Intra-prefrontal 8-OH-DPAT and M100907 improve visuospatial attention and decrease impulsivity on the five-choice serial reaction time task in rats. Psychopharmacology 167:304-314. 